\title{
INFLUÊNCIA DE DIFERENTES COEFICIENTES DE DESAGREGAÇÃO NA DETERMINAÇÃO DE EQUAÇÕES IDF PARA AQUIDAUANA/MS
}

\author{
Influence of different breakdown coefficients in the \\ determination of IDF equations for Aquidauana/MS
}

\author{
Paulo Eduardo Teodoro', Matheus Piazzalunga Neivock², José Roberto Franco Marques ${ }^{3}$, \\ Amon Micael Fernandes Flores ${ }^{4}$ e Cátia Cristina Braga Rodrigues ${ }^{5}$
}

Recebido em 07 de março de 2014; recebido para revisão em 29 de abril de 2014; aceito em 18 de maio de 2014; disponivel on-line em 08 de outubro de 2014.

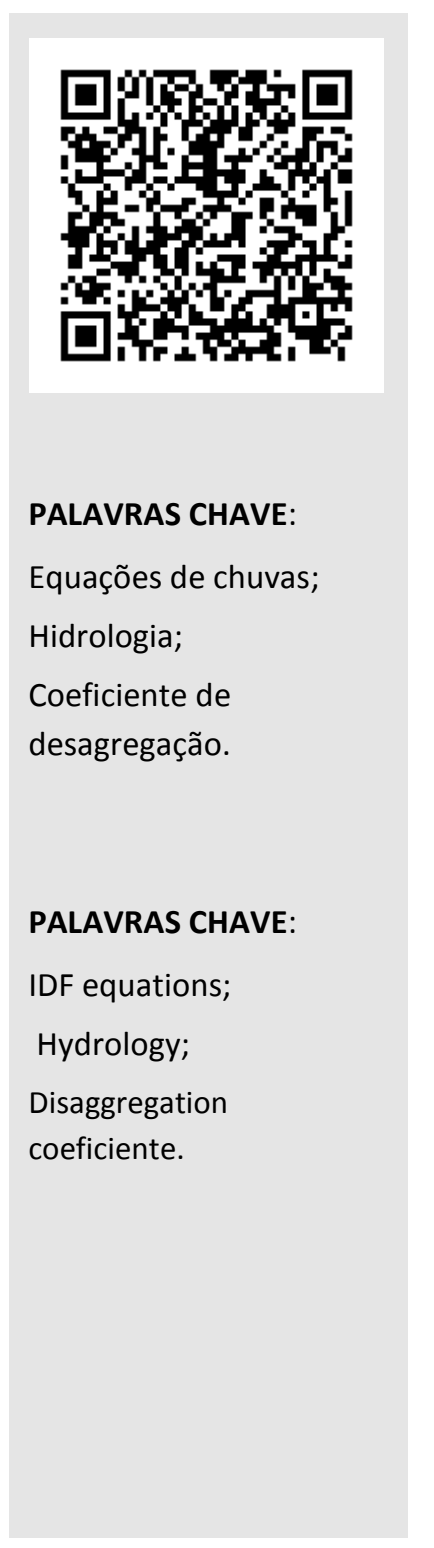

\begin{abstract}
RESUMO: Na literatura são encontradas diversas pesquisas sobre a desagregação de chuvas diárias para determinação de equações de Intensidade-Duração-Frequência (IDF). Entretanto, ainda não existe um trabalho que avaliou a influência de diferentes coeficientes de desagregação na determinação de equações IDF para uma localidade. Assim, objetivou-se com esta pesquisa desenvolver equações de chuvas intensas (curvas IDF) para o município de Aquidauana/MS por meio do emprego de diferentes metodologias de desagregação de chuvas diárias e verificar a influência dos coeficientes utilizados. Foram obtidas as séries de chuvas diárias entre os anos de 2001 a 2013. Foram separados os valores máximos de precipitação para cada ano. Posteriormente, foram aplicados, a cada valor de precipitação os diferentes coeficientes de desagregação de chuvas diária. Verificou-se o ajuste da distribuição de Gumbel aos dados amostrais pelo teste de aderência de Kolmogorov-Sminorv e pelo coeficiente de determinação. Calcularam-se então os valores de precipitação para os períodos de retorno utilizados em projetos de obras hidrológicas: 2, 5, 10, 25, 50 e 100 anos. Para cada período de retorno e com as durações estabelecidas foram determinadas as intensidades máximas médias. De posse das séries de intensidades foram calculados os parâmetros da Equação IDF, via método de regressão linear, logaritmizando-a. Constatou-se que os coeficientes de desagregação de chuvas diárias influenciam consideravelmente na determinação dos parâmetros da equação IDF. As equações IDF determinadas nesta pesquisa poderão contribuir significativamente para o dimensionamento de projetos hidráulicos e de saneamento no município de Aquidauana/MS.
\end{abstract}

ABSTRACT: Several studies in the literature on disaggregation of daily rainfall for determining equations of intensity-duration-frequency (IDF) are found. However, there is still no study evaluated the influence of different factors in determining the breakdown of IDF equations for a location. Thus, the objective of this research was to develop equations to heavy rains (IDF curves) for the municipality of Aquidauna/MS by employing different methods of disaggregation of daily rainfall and the influence of the coefficients used. The series of daily rainfall between the years 2001-2013. Maximum precipitation values for each year were separated. Subsequently, were applied to each value of the different coefficients rainfall of disaggregation daily rainfall. Found to fit the Gumbel's distribution to sample data by the Kolmogorov-Sminorv's adherence and the coefficient of determination. Then calculated the values of precipitation for return periods used in the design of hydraulic structures: 2, 5, 10, 25, 50 and 100 years. For each return period and the durations were established certain average maximum intensities. Possession of intensities series parameters were calculated from IDF equation, by linear regression method, logaritmizando it. It was found that the coefficients of disaggregation of daily rainfall significantly influence the determination of the parameters of the IDF equation. The IDF equations determined in this study may contribute significantly to the design of hydraulic and sanitation projects in the municipality of Aquidauna/MS.

\footnotetext{
* Contato com os autores: página seguinte
} 
* Contato com os autores:

${ }^{1}$ e-mail : eduteodoro@uniderp.edu.br (P.E.Teodoro)

Discente do curso de Engenharia Civil da Universidade Anhanguera-UNIDERP - Campo Grande / MS

²e-mail : neivock@uniderp.edu.br ( M. P. Neivock)

Engenheiro de Materiais, Mestre em Engenharia de Materiais e Modelagem Matemática, docente do curso de Engenharia Civil da Universidade Anhanguera-UNIDERP - Campo Grande / MS.

${ }^{3}$ e-mail : jroberto@uniderp.edu.br (J.R. F. Marques )

Engenheiro Civil, Mestre em Fenômenos dos Transportes, docente do curso de Engenharia Civil da Universidade Anhanguera UNIDERP - Campo Grande / MS

${ }^{4}$ e-mail : amon@uniderp.edu.br ( A. M. F. Flores )

Engenheiro de Civil, Mestre em Tecnologias Ambientais e Recursos Hídricos, docente do curso de Engenharia Civil da Universidade Anhanguera-UNIDERP - Campo Grande / MS.

${ }^{5}$ e-mail : catcrisrodrigues@gmail.com (C. C. B. Rodrigues )

Meteorologista, Mestrado em meteorologia, meteorologista do Centro de Monitoramento de Tempo, do Clima e dos Recursos Hídricos de Mato Grosso do Sul (Centec/AGRAER).

ISSN: 2179-0612

\section{INTRODUÇÃO}

A ocorrência de inundações nas áreas urbanas e rurais em diversas partes do Brasil e do mundo tem aumentado devido às ações antrópicas e às mudanças climáticas que afetam a intensidade das chuvas (MENDES e MEDIONDO, 2007). No meio urbano levam à destruição de bens e perdas humanas (MCT/CGE, 2002). Além disto, em áreas rurais as chuvas intensas e as inundações removem o solo superficial, reduzem sua produtividade, transportam os sedimentos para os corpos hídricos e comprometem sua qualidade e capacidade de armazenamento (SANTOS et al., 2010).

Para mitigar os efeitos das inundações, as obras hidráulicas (vertedores, canais, barragens e sistemas de drenagem) são dimensionadas com base na vazão máxima (Teixeira et al., 2011), seja esta observada estatisticamente ou com base no emprego de modelos chuva-vazão (MELLO et al., 2003; DAMÉ et al., 2010). Em pequenas bacias o método racional, que depende da intensidade máxima da chuva (Teixeira et al., 2011) é amplamente utilizado. A intensidade máxima de uma chuva é obtida a partir da relação intensidadeduração-frequência - IDF (Garcia et al., 2011) e sua determinação depende de registros de dados de precipitação cuja duração nem sempre é disponível, haja vista que precisam de análise cuidadosa (SVENSSON et al., 2007).

Alternativamente, dados diários podem ser desagregados a partir de fatores de proporcionalidade (CETESB, 1986; Garcia et al., 2011) ou utilizados via método de Bell (Mello et al., 2003) que se baseia na similaridade entre os mecanismos de tormenta cujos resultados podem ser generalizados. Conforme resultados obtidos por Mello et al. (2003), Oliveira et al. (2008) e Back (2009), dados desagregados se ajustam melhor aos das relações IDF ajustadas com dados de pluviogramas do que as obtidas com o método de Bell.

Neste sentido, o presente trabalho tem como objetivo avaliar a influência de diferentes metodologias de desagregação de chuvas diárias na determinação de equações de chuvas intensas (curvas IDF) para o município de Aquidauana/MS.

\section{REFERENCIAL TEÓRICO}

O conhecimento das características da precipitação e sua relação no ciclo hidrológico apresenta elevada importância para estudos estratégicos associados ao planejamento do meio ambiente (Carvalho e Assad, 2005), à geração de energia e ao manejo da agricultura, especialmente em condições tropicais (Mello et al., 2003). Um exemplo clássico de sua utilização é no dimensionamento de obras de drenagem superficial, onde é necessário conhecer a IDF da precipitação a ser utilizada no projeto (Back et al., 2012). De acordo com estes autores, a utilização de equações de chuvas intensas é a forma mais usual para estimar a intensidade da chuva a ser aplicada na determinação de vazões de cheia. 
No Brasil existe relativa facilidade em obter dados de precipitação diária, porém dados com menor duração são escassos, devido à falta de equipamentos registradores e, quando existem, são séries relativamente curtas que apresentam muitas falhas nos registros de dados (Back et al., 2012). Lima et al. (2005), Oliveira et al. (2008) e Back (2009) relataram à dificuldade de obtenção de longas séries de dados de precipitação, principalmente de registros pluviográficos. Cecílio e Pruski (2003) destacam que a metodologia para determinar as relações IDF de chuvas exige exaustivo trabalho para tabulação, análise $\mathrm{e}$ interpretação de uma grande quantidade de dados.

Algumas metodologias foram
desenvolvidas visando à obtenção das chuvas de menor duração, a partir dos dados pluviométricos diários (Back et al., 2012). Bell (1969) apresentou relações para estimar a altura da chuva com duração de até 120 min a partir da chuva com duração de uma hora e período de retorno de 10 anos. Torrico (1975) desenvolveu a metodologia das isozonas, que pode ser aplicada em todo o território nacional. Damé et al. (2006) ajustaram as relações IDF com dados de precipitação estimada por técnicas de desagregação de chuva diária. No Brasil são largamente empregadas às relações entre durações publicadas pela CETESB (1986), como os trabalhos de MELLO et al. (2003), FERREIRA et al. (2005), SOPRANI e REIS (2007), OLIVEIRA et al. (2008) e ARAGÃO et al. (2013).

Para Genovez e Zuffo (2000) as relações entre precipitações de diferentes durações apresentadas, além de utilizarem períodos muito curtos foram obtidas para uma média nacional. Estes autores ressaltam, também, que estes valores devem ser atualizados e/ou avaliados em comparação com outros, por se tratar de um estudo feito há muito tempo, baseado nas curvas IDF para 98 localidades do Brasil obtidas por Pfafstetter (1957), que utilizou períodos de dados muito curtos sendo, para a maioria dos locais, da ordem de 10 anos de dados pluviográficos.

$\mathrm{Na}$ literatura são encontrados alguns trabalhos (Silveira, 2000; Back et al. 2012) que propõe coeficientes de desagregação alternativos aos utilizados pela CETESB (1986). Entretanto, ainda não foi realizado nenhum trabalho que verifique a magnitude das diferenças entre equações IDF obtidas por cada metodologia, justificando a presente pesquisa.

\section{CARACTERIZAÇÃO DA PRECIPITAÇÃO DO MUNICÍPIO DE AQUIDAUANA/MS.}

A precipitação pluviométrica do município de Aquidauana/MS (Figura 1) apresenta grande variabilidade, sendo que o maior volume desse elemento resulta de processos de meso e macro escala, principalmente de invasões da frente Polar Atlântica, pois a localização da cidade propicia ser um campo de alternância entre sistemas tropicais e polares. A sazonalidade das chuvas na região mostra maior concentração de precipitação acumulada nos meses de dezembro, janeiro, fevereiro e março que representam o verão, podendo chegar a 49,2 \% da precipitação média anual. $O$ período com os maiores índices de precipitações pluviométricas se relaciona à primavera-verão, onde as temperaturas estão mais elevadas ultrapassando a marca dos $30{ }^{\circ} \mathrm{C}$ nos meses de fevereiro, março, outubro e dezembro (ARTIGAS e ANDRADE, 2011).

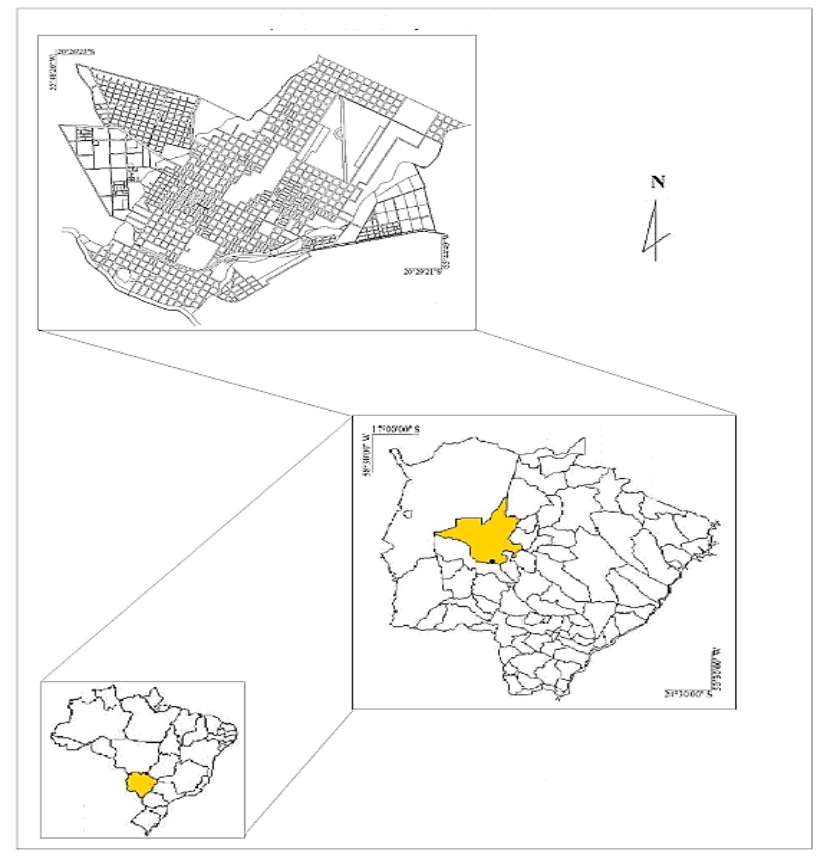

FIGURA 1. Localização do município de Aquidauana/MS. FONTE: ARTIGAS (2010). 
Ainda de acordo com Artigas e Andrade (2011), a sazonalidade da precipitação neste município tem gerado enchentes e alagamentos frequentes, ocasionando inúmeros danos à população, sobretudo nos anos de 1990, 1997, 2001, 2006 e 2010.

\section{MATERIAL E MÉTODOS}

A intensidade de uma precipitação qualquer é a relação entre a altura precipitada e sua duração. Para fins de estimativa da vazão máxima, a intensidade máxima da precipitação é a variável de interesse, sendo esta referida ao tempo de concentração da bacia e associada a um período de retorno (ARAGÃO et al., 2013). Esta intensidade pode ser obtida mediante as relações IDF (Equação 1), estabelecidas a partir de registros de chuva e duração advindos de dados pluviógraficos (SILVA et al., 2002).

$$
i=\frac{K \cdot T_{r}^{a}}{(t+B)^{c}}
$$

Onde:

$\mathbf{i}=$ intensidade máxima, em $\mathrm{mm} \mathrm{h}^{-1}$;

$\mathbf{T}_{\mathbf{r}}=$ período de retorno, em anos;

$\mathbf{t}=$ duração da chuva, em minutos;

a, B, c, K = parâmetros a determinar.

Porém, para um determinado local a determinação dos parâmetros desta equação requer informações de intensidade de chuvas, geralmente escassas (ARAGÃO et al., 2013). Na ausência desses dados é utilizado o método da desagregação de chuva diária (Damé et al., 2010) que relaciona a chuva de 1 dia (observada em pluviômetro) com a chuva de $24 \mathrm{~h}$ (obtida no pluviógrafo) e desta para tempos menores por meio do emprego de alguns coeficientes encontrados na literatura (Tabela 1). Paralelamente foi considerado, para cada duração citada, um valor limite mínimo do total precipitado a partir do qual a chuva pode ser considerada intensa (Tabela 2), sendo eliminados das séries os valores inferiores (SILVA, 2009).

\section{TABELA 1: Coeficientes de desagregação para diferentes durações de chuva.}

\begin{tabular}{cccc}
$\begin{array}{c}\text { Relação entre alturas } \\
\text { pluviométricas }\end{array}$ & \multicolumn{3}{c}{ Coeficiente de desagregação } \\
\hline 5 min para 30 min & 0,340 & Silveira (2000) & Back et al. (2012) \\
10 min para 30 min & 0,540 & ---- & 0,350 \\
15 min para 30 min & 0,700 & ---- & 0,530 \\
30 min para $1 \mathrm{~h}$ & 0,740 & ---- & 0,680 \\
5 min para $24 \mathrm{~h}$ & ----- & 0,750 \\
10 min para $24 \mathrm{~h}$ & ---- & ---104 & --- \\
15 min para $24 \mathrm{~h}$ & ---- & 0,177 & ---- \\
30 min para $24 \mathrm{~h}$ & ---- & 0,226 & ---- \\
$1 \mathrm{~h}$ para $24 \mathrm{~h}$ & 0,420 & 0,318 & 0,350 \\
$2 \mathrm{~h}$ para $24 \mathrm{~h}$ & 0,480 & 0,420 & 0,460 \\
$4 \mathrm{~h}$ para $24 \mathrm{~h}$ & ----- & 0,531 & 0,550 \\
$6 \mathrm{~h}$ para $24 \mathrm{~h}$ & 0,720 & 0,651 & 0,610 \\
$8 \mathrm{~h}$ para $24 \mathrm{~h}$ & 0,780 & 0,724 & 0,660 \\
$12 \mathrm{~h}$ para $24 \mathrm{~h}$ & 0,850 & 0,778 & 0,760 \\
$18 \mathrm{~h}$ para $24 \mathrm{~h}$ & ----- & 0,856 & 0,890 \\
$24 \mathrm{~h}$ para 1 dia & 1,140 & 0,936 & 1,160 \\
\hline
\end{tabular}


TABELA 2: Valores mínimos de precipitação adotados.

\begin{tabular}{ccccccccccccc} 
Duração $(\mathrm{min})$ & 5 & 10 & 15 & 30 & 60 & 120 & 240 & 360 & 480 & 720 & 1440 \\
Precipitação adotada $(\mathrm{mm})$ & 8 & 10 & 15 & 20 & 25 & 30 & 35 & 40 & 40 & 47 & 50 & 50 \\
\hline
\end{tabular}

Para o emprego do método da desagregação de chuva faz-se necessário separar, inicialmente, os valores máximos de precipitação para cada ano da localidade questão. Posteriormente, foram aplicados, a cada valor de precipitação, diferentes coeficientes listados na Tabela 1 gerando, assim, séries de precipitação para diferentes durações. Com base nos valores da Tabela 2 foram eliminados da série os valores de precipitação abaixo de $8 \mathrm{~mm}$ com duração de $5 \mathrm{~min}$ (ARAGÃO et al., 2013).

Com relação a séries históricas, estas foram obtidas através do site da Agência Nacional de Águas (ANA, 2014), seguindo-se a recomendação da CETESB (1986), onde o número de anos de dados climáticos para esta análise foi de 13 anos (20012013).

Neste trabalho foram aplicados, utilizando-se planilha eletrônica, os coeficientes listados nas Tabelas 1 e 2 aos dados de séries anuais. Além disto, para Naghettini e Pinto (2007) em pesquisas sobre 0 dimensionamento de projetos hidrológicos, constataram que além da determinação das máximas precipitações observadas nas séries históricas, faz-se necessário também prever, com o auxílio de probabilidades, a frequência associada a essas precipitações. Neste sentido, a distribuição de Gumbel se ajusta satisfatoriamente às distribuições de valores extremos de grandezas hidrológicas, tais como totais precipitados e vazões observadas (Naghettine e Pinto, 2007; Sansigolo, 2008; Ben-Zvi, 2009) sendo, assim, empregada nesta pesquisa.

Conforme Aragão et al. (2013), a distribuição de Gumbel apresenta a seguinte função cumulativa de probabilidade (Equação 2):

$$
F C P: P[Y \leq y]=e^{-e^{-\alpha(y-\mu)}}
$$

Onde:

$F C P=$ função cumulativa de probabilidade;

$P=$ probabilidade de não excedência;

$\alpha, \mu=$ parâmetros da distribuição;

$y=$ variável reduzida.

A variável $\alpha$ corresponde ao parâmetro de escala e $\mu$ o parâmetro de locação. Por meio do método dos momentos e com as estimativas amostrais " $x$ " (média aritmética) e "S" (desvio padrão), tem-se, como resultado as Equações 03 a 06 :

$$
\begin{array}{cc}
z=\alpha(y-\mu) & \text { Eq.[3] } \\
\alpha=\frac{1,2826}{S} & \text { Eq.[4] } \\
\mu=\bar{x}-0,451 S & \text { Eq.[5] } \\
y=\left[x-\bar{x}+S\left(\frac{\bar{y}_{n}}{S_{n}}\right)\right] \frac{S_{n}}{S} & \text { Eq.[6] }
\end{array}
$$

Onde:

$\mathbf{y}_{\mathbf{n}}$ e $\mathbf{S}_{\mathbf{n}}$ são respectivamente iguais à média e ao desvio padrão da variável reduzida.

A fim de verificar se a distribuição de Gumbel se ajustou de forma correta ou não aos dados amostrais, compararam-se as frequências amostrais com as frequências teóricas esperadas pelo teste de aderência de Kolmogorov-Sminorv (KS) a nível de significância de $5 \%$ e pelo coeficiente de determinação $\left(R^{2}\right)$.

O teste KS é um teste não paramétrico (ou seja, independe dos parâmetros da distribuição, ou uma estimativa destes, para o cálculo de sua estatística), e tem como base a diferença máxima entre as funções de probabilidades acumuladas, empírica e teórica, de variáveis aleatórias contínuas. Este teste não é aplicável a variáveis aleatórias discretas (Martins et al., 2011). Este teste é comum no uso de análises de precipitação 
de chuvas intensas (OLIVEIRA et al., 2008; MARTINS et al., 2011; ALVES et al., 2013; ARAGÃO et al., 2013).

Após verificação do ajuste da distribuição de Gumbel às séries desagregadas, calcularam-se então os valores de precipitação para os períodos de retorno utilizados em projetos de obras hidráulicas (CETESB, 1986): 2, 5, 10, 25, 50, 100 anos. Para cada período de retorno e com as durações da Tabela 2 foram determinadas as intensidades máximas médias. De posse das séries de intensidades foram calculados os parâmetros da Equação 1, via método de regressão linear, logaritmizando-a (NAGHETTINI e PINTO, 2007; SANSIGOLO, 2008; ARAGÃO et al., 2013).

Assim, foram geradas três equações IDF para cada localidade, em função de cada família de coeficientes de desagregação. Os resultados obtidos pelas equações ajustadas pelos coeficientes de Silveira (2000) e Back et al. (2012) foram comparados com os obtidos pelos coeficientes da CETESB (1986), por meio do erro padrão de estimativa (EPE), conforme recomendações de ARAGÃO et al. (2013) (Equação 7).

$$
E P E=\sqrt{\frac{\left(\frac{i_{m}^{C E T E S B}-S / B}{i_{m}^{S / B}}\right)^{2}}{N}}
$$

Onde:

$i_{m}^{S / B}=$ intensidade máxima média de precipitação obtida pela equação ajustadas pelos coeficientes de Silveira (2000) e Back et al. (2012);

$i_{m}^{\text {CETESB }}=$ intensidade máxima média de precipitação obtida pela equação ajustada pelos coeficientes da CESTESB (1986);

$N$ = número de durações.

\section{RESULTADOS E DISCUSSÃO}

O teste KS aceitou a distribuição de Gumbel para todas as durações, independente do coeficiente de desagregação utilizado (Tabela 3). Isto confirma os resultados encontrados na literatura sobre as distribuições de probabilidades aplicadas a valores extremos máximos, em que a distribuição de Gumbel (Naghettine e Pinto, 2007; Sansigolo, 2008; Ben-Zvi, 2009) surge como a mais indicada e melhor ajustada. Além disto, estes resultados corroboram a afirmação de Alves et al. (2013) de que 13 eventos são suficientes para estimar os parâmetros das distribuições quando testado sua aderência pelo teste KS.

TABELA 3: Valores de D no teste de aderência de Kolmogorov-Sminorv.

\begin{tabular}{|c|c|c|c|}
\hline Tempo de precipitação & CETESB (1986) & Silveira (2000) & Back et al. (2012) \\
\hline 5 minutos & 0,2346 & 0,2288 & 0,2341 \\
\hline 10 minutos & 0,2022 & 0,1993 & 0,2018 \\
\hline 15 minutos & 0,2341 & 0,2199 & 0,2337 \\
\hline 30 minutos & 0,2211 & 0,2121 & 0,2212 \\
\hline 1 hora & 0,1585 & 0,1505 & 0,1586 \\
\hline 2 horas & 0,2022 & 0,1982 & 0,2020 \\
\hline 4 horas & 0,1771 & 0,1671 & 0,1770 \\
\hline 6 horas & 0,1833 & 0,1793 & 0,1834 \\
\hline 12 horas & 0,1791 & 0,1651 & 0,1790 \\
\hline 18 horas & 0,1851 & 0,1703 & 0,1850 \\
\hline 24 horas & 0,1831 & 0,1670 & 0,1829 \\
\hline
\end{tabular}


Os valores de $\mathrm{D}$ obtidos pelo teste KS não apresentaram diferenças em magnitudes elevadas para os diferentes coeficientes de desagregação avaliados. Os valores críticos D para o este teste, a um nível de confiança de $95 \%$ e, tamanho da série igual a 13 é 0,361 , sendo que um valor elevado da estatística revela grandes diferenças entre as frequências observadas e esperadas, apresentandose como um indicador da pouca aderência da distribuição em estudo. Desta forma, apesar de pequena diferença, os valores propostos por Silveira (2000) para a desagregação de chuvas diárias apresentaram maior aderência à distribuição de Gumbel ( $D$ mais próximo à zero), fato este, confirmado pelo maior coeficiente de determinação obtido (Tabela 4).

Os parâmetros da equação IDF determinados com os coeficientes de desagregação recomendados por Silveira (2000) não apresentaram grande diferença em relação à equação que utilizou os coeficientes da CETESB (1986), apresentando EPE de 1,59\%.

Os coeficientes de desagregação de chuva diária recomendados por Back et al. (2012) influenciaram consideravelmente na determinação dos parâmetros da equação IDF, em relação aos parâmetros determinados com os coeficientes da CETESB (1986), apresentando EPE de 4,73\%. Esta maior variação pode ter ocorrido porque o estudo de Back et al. (2012) incluiu dados mais recentes de chuvas intensas no estado de Santa Catarina. Entretanto, de acordo com Silveira (2000), os coeficientes determinados pela CETESB (1986) são, notavelmente, semelhantes para regimes pluviométricos diversos do Brasil, por isto não se faz, usualmente, uma diferenciação climatológica regional.

Segundo Villela e Mattos (1975) e Oliveira e Pruski (1985), na seleção do TR para estimativa da intensidade de chuvas a ser empregada na elaboração de projetos de drenagem de superfície, devem-se considerar os custos da obra, o grau de risco, a vida útil da obra, o tipo de estrutura e a facilidade de reparo e ampliação da obra. Desta forma, utilizando os parâmetros da equação determinada com os coeficientes de desagregação da CETESB (1986), determinaram-se os valores de intensidade para projetos de obras e soluções hidrológicas com adoção de parâmetro de períodos de retorno de 2, 5, 10, 25, 50 e 100 anos (Tabela 5).

TABELA 4: Valores dos parâmetros das equações IDF em função de diferentes coeficientes de desagregação.

\begin{tabular}{|c|c|c|c|c|c|c|}
\hline \multirow{2}{*}{ Coeficiente de desagregação } & \multicolumn{4}{|c|}{ Parâmetros da equação IDF } & \multirow{2}{*}{$\mathbf{R}^{2}$} & \multirow{2}{*}{$\begin{array}{l}\text { EPE } \\
\text { (\%) }\end{array}$} \\
\hline & K & a & b & c & & \\
\hline CETESB (1986) & 733,29 & 0,2239 & 7,00 & 0,7047 & 0,964 & ---- \\
\hline Silveira (2000) & 782,93 & 0,1991 & 11,00 & 0,7579 & 0,970 & 1,59 \\
\hline Back et al. (2012) & 453,94 & 0,2067 & 6,00 & 0,6832 & 0,968 & 4,73 \\
\hline Média & 656,72 & 0,2099 & 8,00 & 0,7153 & 0,967 & ---- \\
\hline Desvio padrão & 177,36 & 0,0127 & 2,65 & 0,0385 & 0,003 & ----- \\
\hline
\end{tabular}


TABELA 5: Intensidade de chuvas ( $\mathrm{mm} / \mathrm{h}$ ) em função do período (anos) de retorno e duração (minutos).

\begin{tabular}{ccccccc}
\hline $\begin{array}{c}\text { Duração } \\
\text { (minutos) }\end{array}$ & $\mathbf{2}$ & $\mathbf{5}$ & $\mathbf{1 0}$ & $\mathbf{2 5}$ & $\mathbf{5 0}$ & $\mathbf{1 0 0}$ \\
\cline { 2 - 7 } & 135,13 & 187,80 & 222,68 & 266,75 & 299,44 & 331,89 \\
$\mathbf{5}$ & 103,09 & 143,27 & 169,88 & 203,49 & 228,43 & 253,19 \\
$\mathbf{1 0}$ & 89,09 & 123,81 & 146,81 & 175,86 & 197,41 & 218,80 \\
$\mathbf{1 5}$ & 63,63 & 88,44 & 104,86 & 125,61 & 141,01 & 156,29 \\
$\mathbf{3 0}$ & 43,00 & 59,76 & 70,85 & 84,87 & 95,28 & 105,60 \\
$\mathbf{6 0}$ & 24,57 & 34,15 & 40,49 & 48,50 & 54,44 & 60,34 \\
$\mathbf{1 2 0}$ & 15,36 & 21,34 & 25,30 & 30,31 & 34,03 & 37,71 \\
$\mathbf{2 4 0}$ & 12,28 & 17,07 & 20,24 & 24,25 & 27,22 & 30,17 \\
$\mathbf{3 6 0}$ & 7,25 & 10,08 & 11,95 & 14,31 & 16,07 & 17,81 \\
$\mathbf{7 2 0}$ & 5,66 & 7,86 & 9,33 & 11,17 & 12,54 & 13,90 \\
$\mathbf{1 0 8 0}$ & 4,86 & 6,76 & 8,01 & 9,60 & 10,78 & 11,94 \\
$\mathbf{1 4 4 0}$ & & & & &
\end{tabular}

\section{CONCLUSÕES}

Os coeficientes de desagregação de chuvas diárias influenciam consideravelmente na determinação dos parâmetros da equação IDF, onde os valores propostos por Silveira (2000) e Back et al. (2012) apresentaram 1,59 e 4,53\%, respectivamente de erro de padrão de estimativa em relação aos da CETESB (1986).

As equações IDF determinadas neste trabalho poderão contribuir significativamente para o dimensionamento de projetos hidrológicos no município de Aquidauana/MS. Entretanto, recomenda que estudos de mesma ordem sejam posteriormente realizados para se obter resultados ainda mais satisfatórios.

Ademais, sugere-se que haja ampliação da rede de monitoramento hidrométrica na região, para que novos estudos confirmem os valores propostos dos coeficientes de desagregação.

\section{REFERÊNCIAS BIBLIOGRÁFICAS}

ALVES, A. V. P. et al. Análise de métodos para estimativa dos parâmetros das distribuições de Gumbel e GEV em eventos de precipitações máximas na cidade de CuiabáMT. Revista Eletrônica de Engenharia Civil, Vol. 6, n. 1, 2013, p. 32-43.

ANA - Agência Nacional das Águas. Hidro Web: sistemas de informações hidrológicas. Disponível em: <http://hidroweb.ana.gov.br>. Acessado em 10 de Janeiro de 2014.
ARAGÃO, R. et al. Chuvas intensas para o estado de Sergipe com base em dados desagregados de chuva diária. Revista Brasileira de Engenharia Agrícola e Ambiental, Vol.17, n. 3, 2013, p.243-252.

ARTIGAS, E. F. Inundações no espaço urbano da cidade de Aquidauana-MS. (Trabalho de Conclusão de Curso). Universidade Federal de Mato Grosso do Sul, UFMS. Aquidauana, MS, 2010. p.243-252.

ARTIGAS, E. F; ANDRADE, V. S. A. A vulnerabilidade espacial climática na cidade de Aquidauana-MS/Brasil. Revista Geográfica de América Central, Vol. 4, n. 3, 2011, p. 1-19.

BACK, A. J. Relações entre precipitações intensas de diferentes durações ocorridas no município de Urussanga, SC. Revista Brasileira de Engenharia Agrícola e Ambiental, Vol. 13, 2009, n. 2 p.170-175.

BACK, A. J. et al. Relações entre precipitações intensas de diferentes durações para desagregação da chuva diária em Santa Catarina. Revista Brasileira de Engenharia Agrícola e Ambiental, Vol. 16, n. 4, 2012, p.391-398.

BELL, F. G. Generalized rainfall-duration-frequency relationships. Journal of Hydraulics Division, Vol. 95, n. 1 , 1969, p311-327.

BEN-ZVI, A. Rainfall intensity-duration-frequency relationships derived from large partial duration series. Journal of Hydrology, Vol. 367, n. 1, 2009, p.104-114.

CARVALHO, J. P.; ASSAD, E. D. Análise espacial da precipitação pluviométrica no Estado de São Paulo: Comparação de métodos de interpolação. Revista Engenharia Agrícola, Vol. 25, n. 2, 2005, p.377-384. 
CECÍLIO, R. A.; PRUSKI, F. F. Interpolação dos parâmetros da equação de chuvas intensas com uso do inverso de potências da distância. Revista Brasileira de Engenharia Agrícola e Ambiental, Vol. 7, n. 3, 2003, p.501-504.

CETESB - Companhia de Tecnologia de Saneamento Ambiental. Drenagem urbana: manual de projeto. 1.ed. São Paulo: DAEE/CETESB, 1986, 466p.

DAMÉ, R. C. F. et al. Comparação entre curvas intensidade-duração-frequência de ocorrência de precipitação obtidas a partir de dados pluviográficos com aquelas estimadas por técnicas de desagregação de chuva diária. Revista Brasileira de Agrociência, Vol. 12, n. 4, 2006, p.505-509.

DAMÉ, R. C. F. et al. Hidrograma de projeto em função da metodologia utilizada na obtenção da precipitação. Revista Brasileira de Engenharia Agrícola e Ambiental, Vol. 14, n. 1, 2010, p.46-54.

FERREIRA, J. C. et al. Parâmetros para equações mensais de estimativas de precipitação de intensidade máxima para o Estado de São Paulo - Fase I. Revista Ciência e Agrotecnologia, Vol. 29, n. 6, 2005, p.1175-1187.

GARCIA, S. S. et al. Determinação da equação intensidade-duração-frequência para três estações meteorológicas do Estado de Mato Grosso. Revista Brasileira de Engenharia Agrícola e Ambiental, Vol. 15, n. 6, 2011, p.575-581.

GENOVEZ, A. M.; ZUFFO, A. C. Chuvas intensas no Estado de São Paulo: Estudos existentes e análise comparativa. Revista Brasileira de Recursos Hídricos, Vol. 5, n. 2000, p.45-58.

LIMA, H. M. F. et al. Aplicação e validação de um simulador estocástico de variáveis climáticas: $O$ caso da precipitação. Revista Ingenieria del Agua, Vol. 12, n. 1, 2005, p.27-37.

MARTINS, J. A. et al. Distribuição das chuvas no município de Tangará da Serra, médio norte do Estado de Mato Grosso, Brasil. Acta Scientiarum. Agronomy, vol. 33, n. 2, 2011, p. 193-200.

MCT/CGE - Ministério de Ciência e Tecnologia/Centro de Estudos e Gestão Estratégica. Diretrizes estratégicas para o Fundo de Recursos Hídricos de Desenvolvimento Científico e Tecnológico. Brasília, 2002. Disponível em <http://www.ana.gov.br> Acessado em 11 de Outubro de 2013.

MELLO, C. R. et al. Modelos matemáticos para predição da chuva de projeto para regiões do Estado de Minas Gerais. Revista Brasileira de Engenharia Agrícola e Ambiental, Vol.7, n. 3, 2003, p.121-128.

MENDES, H. C.; MENDIONDO, E. M. Histórico da expansão urbana e Incidência de Inundações: O Caso da Bacia do Gregório, São Carlos - SP. Revista Brasileira de Recursos Hídricos, Vol.12, n. 1, 2007, p.17-27.
NAGHETTINI, M.; PINTO, E. J. A. Hidrologia estatística. Belo Horizonte: CPRM. 2007, 552p.

OLIVEIRA, L. F. C.; PRUSKI, F. F. Modelos para estimar as perdas de solo e água e transporte de solutos. Viçosa, MG. 1985, 70p.

OLIVEIRA, L. F. C., ANTONINI, J. C. A., FIOREZE, A. P., SILVA, M. A. S. Métodos de estimativa de precipitação máxima para o Estado de Goiás. Revista Brasileira de Engenharia Agrícola e Ambiental, Vol. 12, n. 6, 2008, p.620-625.

PFAFSTETTER, O. Chuvas intensas no Brasil: relação entre precipitação, duração e frequência de chuvas em 98 postos com pluviógrafos. Rio de Janeiro: DNOCS, 1957, 419p.

SANSIGOLO, C. A. Distribuições de extremos de precipitação diária, temperatura máxima e mínima e velocidade do vento em piracicaba, SP (1917-2006). Revista Brasileira de Meteorologia, Vol. 23, n. 3, 2008, p.341-346.

SANTOS, G. S. et al. Chuvas intensas relacionadas à erosão hídrica. Revista Brasileira de Engenharia Agrícola e Ambiental, Vol. 14, n. 2, 2010, p.115-123.

SILVA, B. M. Chuvas intensas em localidades do estado de Pernambuco. Dissertação de Mestrado em Engenharia Civil. Universidade Federal do Pernambuco UFPE, Recife: UFPE, 2009, 100p.

SILVA, D. D. et al. Chuvas intensas no Estado da Bahia. Revista Brasileira de Engenharia Agrícola e Ambiental, Vol. 6, n. 2, 2002, p.362-367.

SILVEIRA, A. L. L. Equação para coeficientes de desagregação de chuva. RBRH - Revista Brasileira de Recursos Hídricos, Vol. 5, n. 4, 2000, p.143-147.

SOPRANI, M. A.; REIS, J. A. T. Proposição de equações de Intensidade-Duração-Frequência de precipitações para a bacia do rio Benevente, ES. Revista Capixaba de Ciência e Tecnologia, Vol. 1, n. 2, 2007, p.18-25.

SVENSSON, C. et al. An experimental comparison of methods for estimating rainfall intensity-durationfrequency relations from fragmentary records. Journal of Hydrology, Vol. 341, n. 3, 2007, p.79-89.

TEIXEIRA, C. F. A. et al. Vazão máxima de projeto: Metodologia para dimensionamento de bueiros em áreas agrícolas. Teoria e Prática na Engenharia Civil, Vol. 17, n. 1, 2011, p.49-56.

TORRICO, J. J. T. Práticas hidrológicas. Rio de Janeiro: Transcon, 1975, 119p.

VILLELA S. M.; A. MATTOS. Hidrologia aplicada. McGrawHill do Brasil, São Paulo, SP. 1975, 245p. 